\title{
Frakturen und Luxationen der oberen Halswirbelsäule
}

\author{
$\square$ Dankward Höntzsch, Andreas Badke, Hans-Peter Kaps
}

\section{Zusammenfassung}

Verletzungen der Halswirbelsäule sind durch die exponierte Lage nicht selten. Das Spektrum reicht von leichten Verletzungen wie Verstauchungen bis zu schweren Luxationsfrakturen. Mit die wesentliche Frage ist, ob eine teilweise oder vollkommene Lähmung vorliegt oder nicht. Bei Vorliegen einer sensiblen oder motorischen Lähmung sind im Regelfall immer die Beine betroffen (Paraplegie), zusätzlich können teilweise oder vollständige Ausfälle der Arme- und Handmuskulatur vorliegen, dann sprechen wir von einer Tetraplegie. Das Lähmungsmuster wird durch die Segmenthöhe der Nervenlähmung im Bereich der Halswirbelsäule bestimmt. Es werden Verletzungen der oberen und unteren Halswirbelsäule unterschieden. Die ersten beiden Halswirbel sind anatomisch unterschiedlich zur Restwirbelsäule und bedürfen auch anderer Therapiekonzepte (z.B. Densverschraubung). Die untere Halswirbelsäule (auch mittlere und untere genannt) reicht vom 3. - 7. Halswirbel. Der Halswirbelsäule wird funktionell und operationstechnisch häufig noch der 1. und 2.Brustwirbel hinzugerechnet. Die obere Halswirbelsäule kann unterteilt werden in den so genannten kraniozervikalen Übergang. Hierbei wird der gelenktragende und gelenkbildende Anteil der Schädelkalotte funktionell zur Halswirbelsäule gerechnet und für das Zervikalsegment mit der Abkürzung $C$ benannt und als Gegenstück zu C1 mit der Ziffer 0 besetzt. Hieraus resultiert die Bezeichnung „CO“. Der kraniozervikale Übergang wird somit mit $\mathrm{CO} / \mathrm{C} 1$ beziffert. Die restliche obere Halswirbelsäule wird mit den Übergängen $\mathrm{C} 1 / \mathrm{C} 2$ und C2/C3 beziffert. Diese Frakturen benötigen eine aufwändige diagnostische Abklärung und ganz spezielle Operationsmethoden. Das ist Grund, warum Frakturen und Luxationen der oberen Halswirbelsäule gesondert beschrieben werden. Für die Behandlung werden konservative und operative Methoden beschrieben. Bei den konservativen Methoden steht nach der Reposition die Ruhigstellung mit Krawatten in leichteren Fällen und mit dem Halofixateur in schwer zu haltenden Fällen. Bei Frakturen und Luxationen, welche erfahrungsgemäß auch bei Ruhigstellung nicht ausreichend sicher ausheilen und welche trotz äußerer Ruhigstellung mit Krawatte oder Halo sich nicht ausreichend stabil einstellen und halten lassen, sind operative Maßnahmen notwendig. Operative Maßnahmen unterscheiden wir in solche von ventral und von dorsal. Diese Operationsmethoden benötigen spezielle anatomische Kenntnisse und Erfahrungen des gesamten Operationsteams betreffend präoperativer Diagnostik, Lagerung, Abdeckung, Instrumenten und Implantaten sowie der Operationsmethode. Diese Komplexität macht möglich, dass speziell für die Behandlung und dann vor allem die operative Versorgung eine interdisziplinäre Teamarbeit zwischen OP-Pflege, operationstechnischer Assistenz und Operateur notwendig ist.

\section{Einleitung}

Die obere Halswirbelsäule umfasst die ersten 2 Halswirbel (C1 und C2) sowie die angrenzenden gelenktragenden Anteile und die Gelenke der Schädelkalotte (CO) und des dritten Halswirbelkörpers (C3).

Die Verletzungsmöglichkeiten, die Diagnostik und die therapeutischen Schritte sind an der oberen Halswirbelsäule sehr vielfältig und komplex.

\section{Literaturhinweis}

Diagnostik und Therapie der oberen Halswirbelsäule sind so komplex und vielfältig, dass in diesem Artikel nur eine Übersicht gegeben werden kann. Für das Studium haben sich folgende Literaturstellen bewährt:

Besonders geeignete und anerkannte Literatur zur weiteren Orientierung von Diagnostik und Therapie der oberen Halswirbelsäule (alphabetisch):

Aebi M, Thalgott JS, Webb JK, AO/ASIF Principles in Spine Surgery. Springer Berlin 1998 (das sog. AO Manual für die Wirbelsäule)

Blauth M. Obere Halswirbelsäule. in Tscherne H, Blauth M (Hrsg.) Tscherne Unfallchirurgie Wirbelsäule. Springer Berlin Heidelberg 1998 („das Standardwerk“ für die Diagnostik und Therapie der Wirbelsäule)

Illgner A, Reilmann H. Verletzungen der Halswirbelsäule. Unfallchirurg 1999; 99 : 351-367 (guter noch heute gültiger Übersichtsartikel)

Rüedi TP, Murphey WM, .... AO Principles of Fracture Management. Thieme Stuttgart 2000 (das sog. „AO Principles“ als Nachfolger des „AO Manuals" $z$. Z. in Englisch; ab 2002 in Deutsch)

OP-Journal Heft 21996 (um auch einen Vergleich und Ergänzung zwischen 2001 und 1996 zu haben)

\section{Anatomie}

Die obere Halswirbelsäule wird gerechnet von der Gelenkfläche der Schädelba- 
sis. Sie wird als CO (Zervikal $=$ Hals 0 ) beschrieben. Weiter beinhaltet der obere Halswirbelsäulenabschnitt den 1. Halswirbel, den sog. Atlas - AiS genannt (C1) und den 2.Halswirbelkörper (C2).

Der erste Halswirbel ist ein Ring, welcher auf dem 2. Halswirbelkörper liegt. In dem Raum, wo die übrigen Wirbel ihren Wirbelkörper haben, ragt der Dens des 2. Halswirbelkörpers. Er ist dort drehbar gelagert. Die vordere Begrenzung ist der knöcherne Atlasbogen, die hintere Begrenzung ein entsprechend stark ausgebildetes Band.

Der 2. Halswirbelkörper besteht aus diesem Dens, welcher den Gelenkstift darstellt. In diesem Gelenk findet die hauptsächliche Drehung des Kopfes statt.

Der Körper und Ring des 2. Halswirbelkörpers ähnelt dann in Anatomie und Funktion den übrigen Halswirbelkörpern. Aus dieser Besonderheit der Funktion der kopfnahen anatomischen Lage und damit auch letztendlich der operationstechnischen Erreichbarkeit wird die obere Halswirbelsäule als besonderer Abschnitt gesehen.

\section{Einteilung der Verletzungstypen}

Wir wollen hier die Verstauchungen und Zerrungen - Distorsion - im Rahmen des OP-Journals nicht näher behandeln. Diese werden konservativ und ambulant therapiert.

Zu diesen Verletzungen zählen auch die sog. „Schleudertraumen“, welche aus vielfältigen Gründen besser als Distorsionen mit der entsprechenden Einteilung benannt werden sollten.

Frakturen und Luxationen können aufgrund folgender Verletzungen eintreten:

Grundsätzlich können verletzt sein:

- knöcherne Strukturen

- Bandstrukturen

- Bandscheiben

- Kombination.

Die Verletzungstypen der oberen Halswirbelsäule können eingeteilt werden in:

- Frakturen der Okzipitalkondylen

- atlantookzipitale Dislokationen

- Frakturen des Atlas

- atlantoaxiale Dislokationen

- Frakturen des Dens axis

- Frakturen des Axiskörpers, seiner Gelenkfortsätze und des Bogens

- kombinierte Verletzungen.

\section{Frakturen der Occipitalkondylen und} atlantooccipitale Dislokationen

Isolierte Frakturen und Verletzungen der Okzipitalkondylen sind selten und schwierig zu diagnostizieren. Sie gehen nahtlos über in Verletzungen der Schädelbasis. Die Therapie wird weitgehend konservativ sein. Anders zu sehen sind Verletzungen des atlantookzipitalen Überganges mit atlantookzipitaler Dislokation.

Die atlantookzipitalen Dislokationen werden nach Blauth [4] wie folgt eingeteilt:

- Typ 1: Ventrale Dislokation, d.h. die Schädelkalotte ist nach vorne disloziert. Es handelt sich um die häufigste Luxation.

- Typ 2: Die dorsale Luxation - selten.

- Typ 3: Axiale Dislokation, d.h. vollkommene Instabilität, Trennung und Längsdislokation des Schädels von der oberen Halswirbelsäule.

- Schwerste Verletzung in den meisten Fällen sofort tödlich.

\section{Diagnostik}

Gute und fein eingestellte Röntgendiagnostik in 2 oder 4 Ebenen, Funktionsaufnahmen auch unter Bildwandlerkontrolle, Computertomogramm.

\section{Empfohlene Therapie [1]}

Notfallmäßig geschlossene Reposition und Ruhigstellung meist im Halofixateur. Es ist darauf zu achten, dass keine zusätzliche Extension ausgeübt wird.

Spätere Behandlung konservativ. Bei Kindern und Jugendlichen und geringer Dis- lokation sowie stabiler Einstellung im Halofixateur.

Operativ bei kompletten überlebten atlantookzipitalen Dislokationen bzw. nicht zu haltenden Situationen mit einer okzipitozervikalen Fusion von der Hinterhauptschuppe auf den 2. und 3. Halswirbelkörper (so kurz wie möglich), d.h. eine sog. CO-C2- oder C3-Fusion im Regelfall mit autologer Spongiosaplastik.

\section{Verletzungen des Atlasbogens (C1)}

Der Atlas kann als Ring verstanden werden. Die Verletzungen können eingeteilt werden in (Abb.1) [8,11]:

- vorne/hinten

- komplett/inkomplett

- stabil/instabil

Wenn der Atlasbogen beidseits gebrochen und der vordere Anteil mit dem anliegenden Dens nach ventral disloziert ist, spricht man von der sog. „Hanged man fracture“ [11].

\section{Verletzungen des 2.Halswirbelkörpers}

- Aufgrund der Anatomie unterscheiden wir in Verletzungen

- des Dens und

- des Körpers mit Bogen und Gelenkfortsätzen.

\section{Verletzungen des Dens axis, sog. Densfrakturen}

Der Dens ist bereits als auf dem Korpus des 2. Halswirbelkörpers stehender Stift beschrieben, welcher dann vom darüberliegenden Halswirbelkörper 1 (Atlas) um-

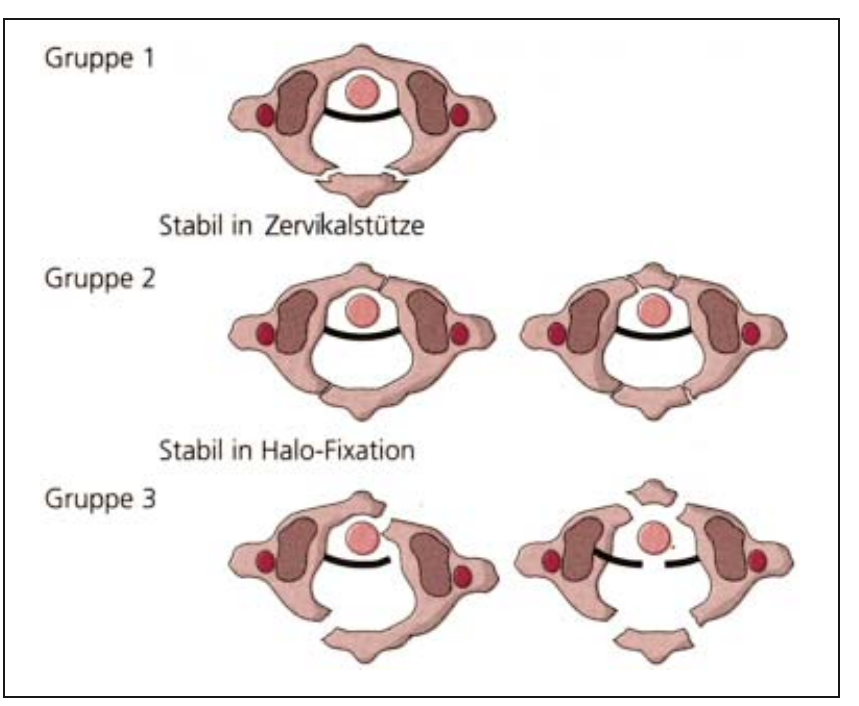

Abb. 1 Einteilung der Frakturen am Ring des Atlas (C1) nach Russe, Bötel, Muhr [10]. 

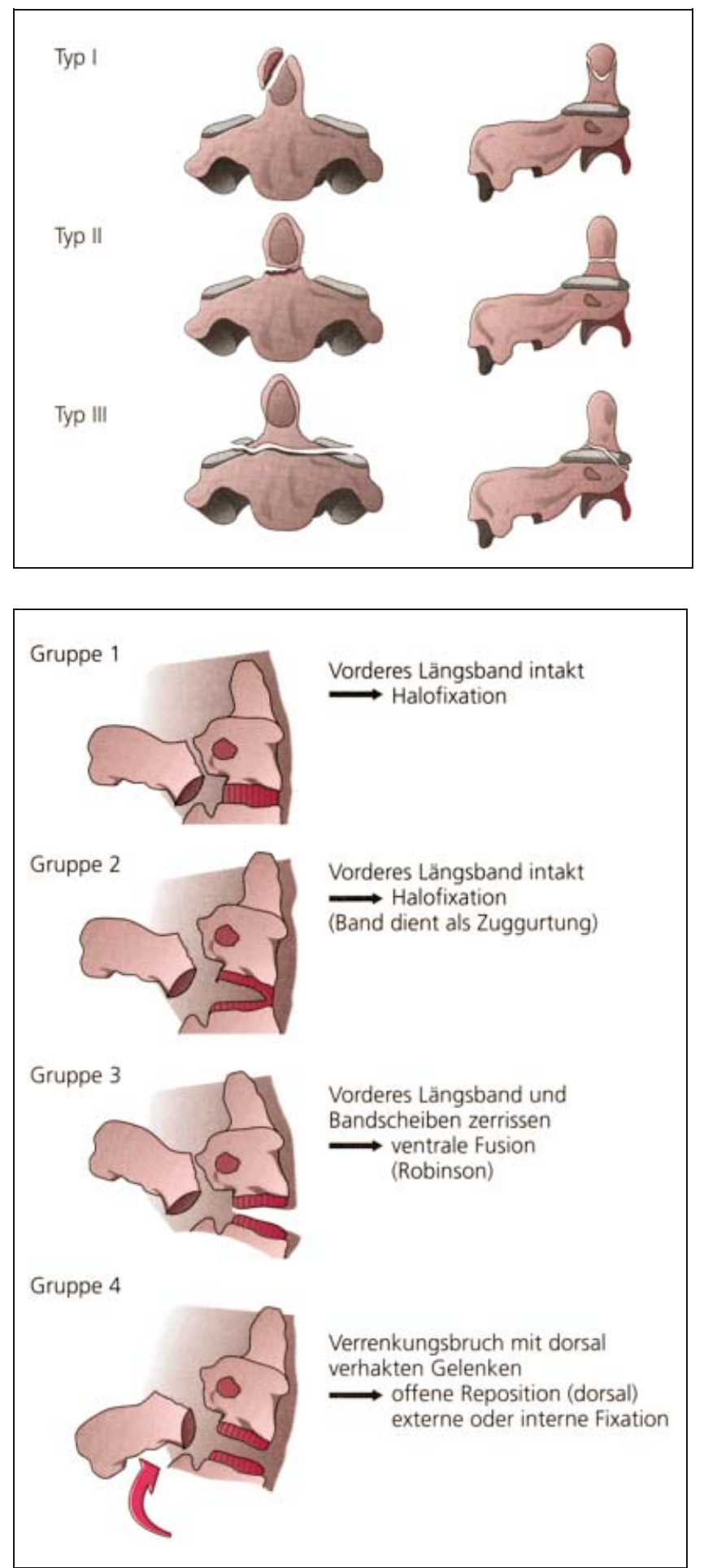

Abb. 2 Einteilung der Densfrakturen, nach Anderson [3]. I Spitzenfrakturen II Frakturen im Schaftbereich, III mehr oder weniger weit in die Basis reichend.

Abb. 3 Einteilung der HWK-II-Ringbrüche mit und ohne Bandverletzung und/ oder Luxation nach Josten, Russe, Hahn, Muhr [8].

schlossen wird: an der Vorderseite von dessen knöchernen Bogen an der Rückseite des Dens von einem entsprechenden Band. In diesem Gelenk wird der Kopf wie in einem Türschanier bewegt. Bei der Densfraktur ist eine Einteilung wie sie Anderson [3] vorgeschlagen hat (Abb. 2) üblich
- I an der Spitze des Dens

- II am Schaft

- III an der Basis, aber mehr oder weniger in den Körper des 2. Halswirbelkörpers hineinreichend.
Die knöcherne Heilung ist um so besser, je weiter die Fraktur in den Korpus hineinreicht. Danach richtet sich auch die Therapie.

\section{Verletzungen des Korpus und des Ringes des Axis (C2)}

Die Einteilung dieser Frakturen wurde sehr gut von Josten und Muhr [8] vorgeschlagen (Abb.3). Die Einteilung richtet sich nach der Verletzung des knöchernen Anteiles sowie der Bandstrukturen, vor allem des vorderen Längsbandes. Hierdurch werden die Dislokation und die mehr oder weniger fehlende Stabilität bestimmt.

Wenn das vordere Längsband erhalten ist und keine Ausrenkung vorliegt, ist die Verletzung als relativ stabil zu betrachten. Kritisch für die Stabilität sind Verletzungen, wo neben einer Fraktur das vordere Längsband verletzt ist. Dies geht meist mit einer kompletten Verletzung der Bandscheibe einher (diskoligamentäre Verletzung). Eine Besonderheit für die Behandlung stellen solche Verletzungen dar, wo es neben der Fraktur, Bandscheiben- und Bandzerreißungen auch $\mathrm{zu}$ einer Verrenkung der Zwischenwirbelgelenke zwischen 2. und 3. Halswirbelkörper gekommen ist (Gruppe 4 nach Josten und Muhr - Abb.3).

\section{Einteilung der verletzten Strukturen}

Die Verletzungen an der oberen Halswirbelsäule können eingeteilt werden wie an der übrigen Halswirbelsäule in:

- reine knöcherne Verletzungen $\rightarrow$ Frakturen

- Verletzungen des Bandapparates $\rightarrow$ ligamentäre Verletzungen

- Bandscheiben $\rightarrow$ diskoligamentäre Verletzungen, (d. h. mit Bandverletzung kombiniert) traumatischer Bandscheibenvorfall

- Ausrenkungen (Luxationen) können vollständig $\rightarrow$ komplette Luxation oder teilweise $\rightarrow$ partielle Luxation oder einseitig $\rightarrow$ einseitige Luxation sein.

- Kombiniert mit einer knöchernen Verletzung spricht man von einer $\rightarrow$ Luxationsfraktur.

\section{Einteilung nach der Nervenverletzung}

Eine weitere wichtige Einteilung ist je nach der Beteiligung des Rückenmarks bzw. der zwischen jedem Wirbelsegment austretenden Nervenwurzel vorzunehmen. Die Einteilung kann nach den motorischen (Beweglichkeit) und sensiblen (Gefühl) Ausfällen beurteilt werden. 

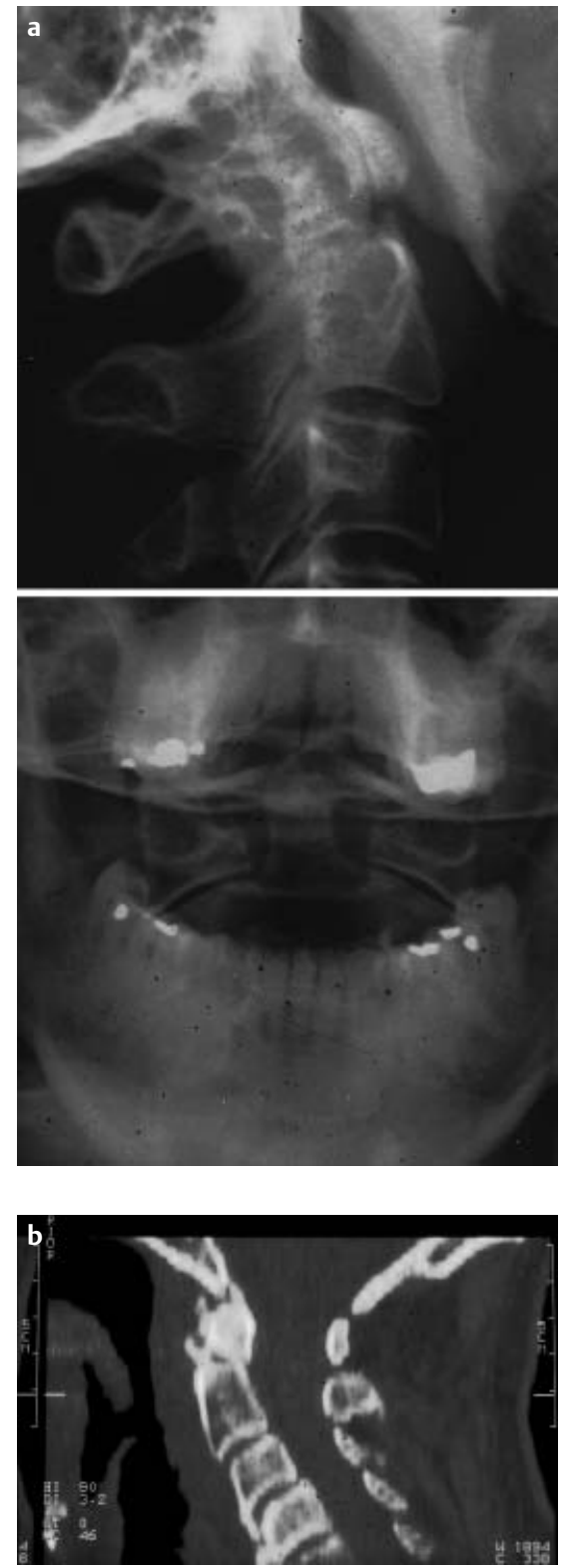

Abb. 4 Densfraktur (a) röntgenologisch in 2 Ebenen (b) im CT.

\section{Diagnostik}

Die diagnostischen Möglichkeiten können erfolgen durch

klinische Untersuchung (in der Reihe des diagnostischen Weges)

neurologischen Untersuchung

Röntgen

- Normalaufnahmen in 2 Ebenen (Abb.4)

- Normalaufnahmen in 4 Ebenen

- Schichtaufnahmen

- Funktionsaufnahmen

Computertomographie (CT)

- Querschnittsbilder

- Rekonstruktionen in Körperlängsebene

- dreidimensionale Rekonstruktionen Kernspintomographie

Die richtigen Diagnoseschritte sind eine hochrangige ärztliche Aufgabe. Zwischen zu viel und zu wenig muss genau abgeschätzt werden. Bei eingetretenen neurologischen Störungen, besonders bei zunehmenden, oder wenn seit dem Unfall nur kurze Zeit vergangen ist, muss insbesondere auch der Zeitfaktor in Rechnung gestellt werden. Die Möglichkeiten und die Auswahl aus dem Diagnosespektrum werden auch durch die gegebenen technischen Möglichkeiten und den Erfahrungsschatz der hinzugezogenen Kollegen bestimmt. Dies alles berücksichtigend muss der Diagnoseweg ganz in den Dienst des Patienten gestellt werden. Neben der Diagnose sollten durch diese Untersuchungen auch der richtige Weg für die Therapie ermöglicht werden.

\section{Therapie}

Die therapeutischen Möglichkeiten können eingeteilt werden in:

- konservativ ohne Ruhigstellung

- konservativ mit Ruhigstellung in Schanzscher Krawatte

- Extensionsbehandlung

- Halo-Fixateur externe

- operativ
Die Distorsionen der Halswirbelsäule und Verletzungen ohne stabilitätsgefährdende Rupturen der Bandstrukturen bis hin zu nicht stabilitätsgefährdenden Knochenfissuren können konservativ behandelt werden. Je nach Schweregrad wird vorübergehend eine weiche oder starre Schanzsche Krawatte verwendet.

Dieses kann in der Regel ambulant durchgeführt werden. Auch diese Behandlung muss sehr differenziert eingesetzt und überwacht werden. Im Rahmen dieser Zusammenstellung für das OP-Journal soll darauf nicht näher eingegangen werden.

\section{Extension (Abb.5)}

Extensionen bewirken, dass am Kopf gegenüber dem Körper gezogen wird.

Extensionen bewirken:

1. Ruhigstellung

2. Reposition

Der Zug am Kopf wird über eine sog. Crutchfield-Klemme erreicht. Hierbei wird der Kopf mit 2 Stiften auf der äußeren Kortikalis wie mit einer Zange gefasst. Diese Crutchfield-Klemmen gibt es in vielfältigen spannbaren Ausführungen. Heute bewähren sich zum Übertragen des Zuges auch Halo-Ringe, die dann später für einen evtl. notwendigen Halo-Fixateur verwendet werden können (s.u.).

Durch den Zug kann eine Ruhigstellung des Kopfes und der Halswirbelsäule gegenüber der Brustwirbelsäule erreicht werden. Zugleich ist auch wie in der übrigen Frakturbehandlung ein Ausrichten von Fragmenten und damit Wiedereingliederung in den normalen Verbund möglich. Hierdurch kann eine Erweiterung eines eingeengten Spinalkanales bewirkt werden. Ein weiteres wesentli-

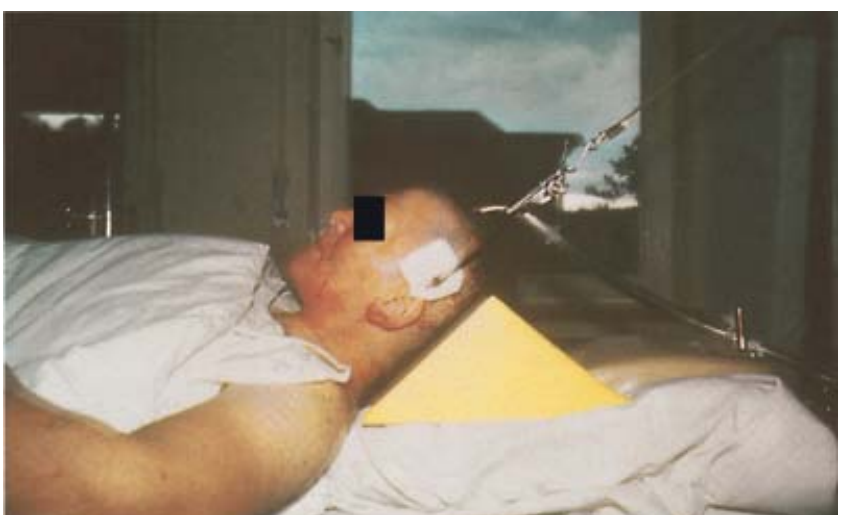

Abb. 5 Patient mit Extension der Halswirbelsäule über Crutchfield-Klemme.
Neurologische Störungen können wie folgt vorliegen:

- ohne neurologische Störung

- komplette Lähmung

- teilweise Lähmung in verschiedenen Formen

- Verletzung einer oder mehrerer Wurzeln

- sich nicht mehr ändernde manifeste Verletzung

- sich verändernde zunehmende/abnehmende Ausfälle

Zur Beurteilung dieser vielfältigen Formen ist eine entsprechende Erfahrung und Ausbildung notwendig. 


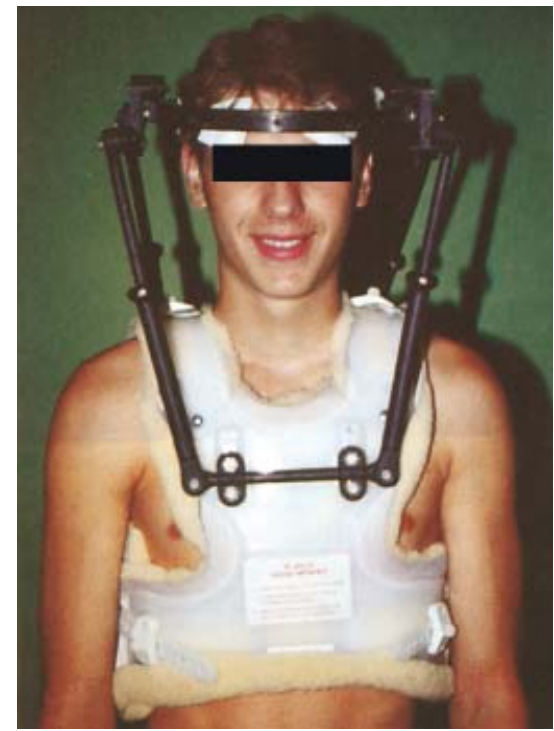

Abb. 6 Stabilisierung der Halswirbelsäule mit Halo-Fixateur externe: am Äquator des Kopfes befestigter Haloring ist über einstellbare (bei neuen Modellen) Kohlefaserstäbe mit einer Brustkorbweste verbunden.

ches Prinzip ist die Reposition von Luxationen. Ausgerenkte Gelenkflächen werden durch Zug soweit auseinandergezogen, dass die korrespondierenden Gelenkränder wie über einen „Berg“ gezogen werden, um dann meist durch Nachlassen des Zuges in die "normale“ Position rutschen zu können. Manchmal sind hierzu mehrere Stunden und aufsteigende Gewichte (bis $20 \mathrm{~kg}$ ) notwendig. Zusätzlich kann durch Veränderung der Zugrichtung (nach vorn oder hinten bzw. zur Seite) Einfluss auf die Reposition genommen werden. Solch ein therapeutisches Vorgehen erfordert die fortlaufende klinische und röntgenologische Kontrolle und fachkundige Überwachung.

Die Extensionsbehandlung ist in der Regel vorübergehend. Dieser Behandlung folgt dann entweder eine Behandlung im Halo-Fixateur externe oder operativ.

\section{Halo-Fixateur externe (Abb.6)}

Der Halo-Fixateur ist eine besondere Form der äußeren Fixation des Kopfes gegenüber dem Brustkorb. Der Kopf wird mit einem Ring gehalten. Der Kopf selbst wird in diesem Ring durch 4 Schrauben zentriert fixiert. Diese Schrauben fassen wie bei der Crutchfield-Klemme nur die äußere Kortikalis der Kopfkalotte in Höhe des größten Durchmessers (Äquators). Die Schrauben können in Lokalanästhesie oder in Allgemeinnarkose ein-
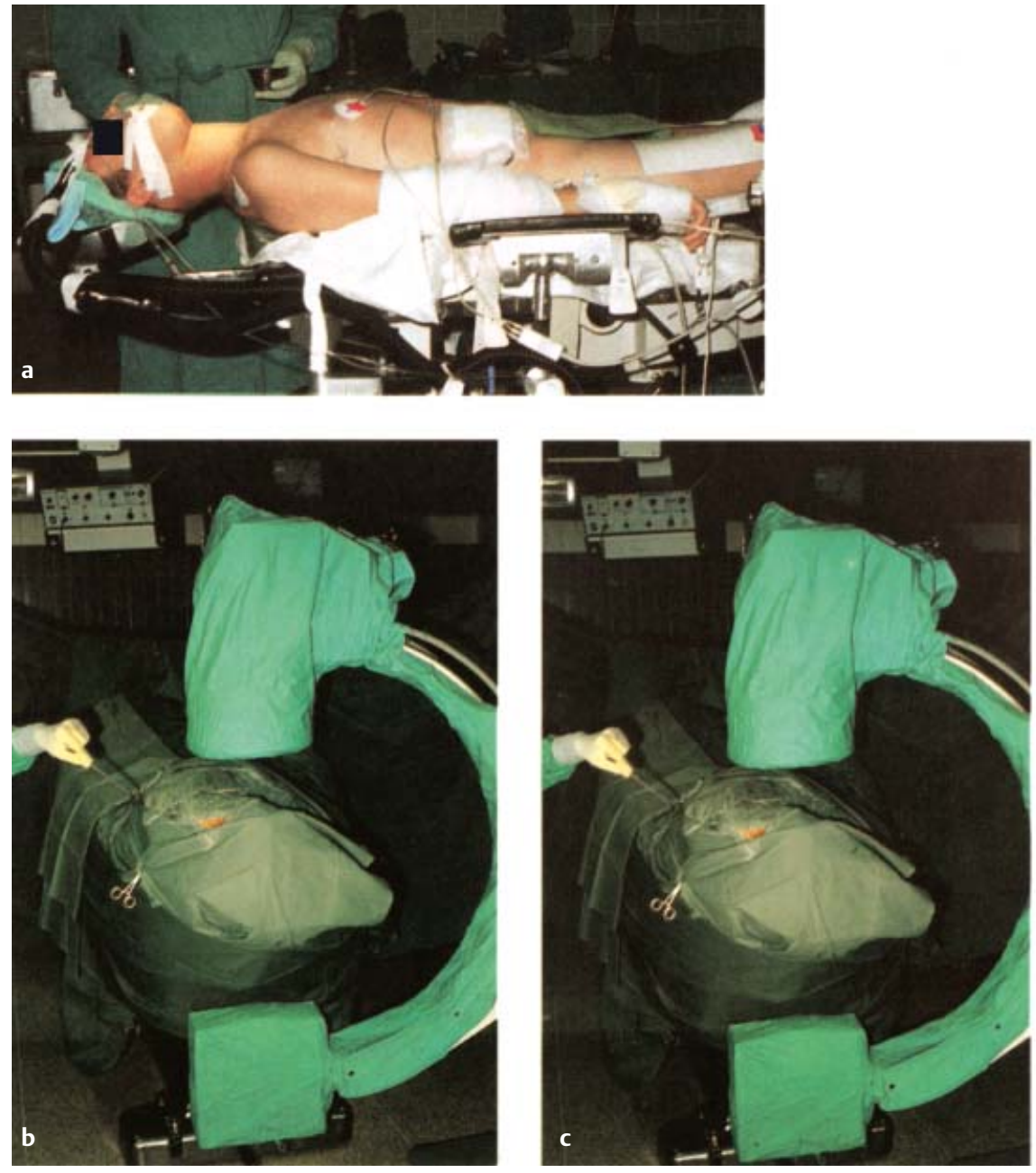

Abb. 7 Lagerung für die operative Versorgung einer Halswirbelsäulenverletzung: Der Kopf liegt in einer Schale oder wird von einem Haloring gehalten, durch Überstrecken (Lordose) wird eine Reposition erzielt. Der Kopf muss über das OP-Tischende so ausgelagert werden, dass die Bildwandlerkontrolle a. p. und seitlich leicht möglich ist. Wir bevorzugen hierzu noch eine sog. Mumienabdeckung (siehe Text).

gebracht werden. Der Ring wird dann über ein besonders einstellbares Stabsystem mit einem gut passenden starren Kunststoffkorsett für den Brustkorb verbunden. Durch Gelenke und ineinander verschiebbare Teleskopstangen ist die Position des Ringes gegenüber dem Brustkorbkorsett frei einstellbar. So kann die Kopfstellung genau ausgerichtet werden. Zusätzlich ist zwischen Kopf und Schultergürtel ein gewisser Zug möglich.

Während des Anlegens des Halo-Fixateurs kommt im Regelfall der Bildwandler zu Anwendung. Zur späteren Kontrolle sind exakte, gut abbildende Röntgenaufnahmen notwenig. Vorteilhafterweise bestehen moderne Halo-Fixateure deshalb aus Kohlefaserringen und -stäben, welche röntgendurchlässig sind.
Der Halo-Fixateur wird, je nach Gesamtverletzung des Patienten und Gegebenheiten des Hauses im Not-OP der Ambulanz und/oder im OP eingesetzt. Beim wachen Patienten kann der Halo im Liegen oder Sitzen angelegt werden. Dies hängt von der Form der Verletzung ab. Beim intubierten Patienten wird der Halo im Regelfall auf dem OP-Tisch angelegt. Die Handhabung der Weste, des Gestelles und des Ringes muss von den behandelnden Ärzten und soweit nötig, assistierenden Pflegekräften einstudiert und geübt sein.

Der Halo-Ring kann auch als Ersatz für die oben beschriebene Crutchfield-Klemme verwendet werden. Dies ist besonders dann von Vorteil, wenn nach der Extension auf ein Halo-Fixateur-externeSystem übergegangen wird. Mancherorts 
hat sich der Halo-Ring auch als Haltevorrichtung des Kopfes für die operative Versorgung von Halswirbelsäulenverletzungen bewährt. Hierfür gibt es auch entsprechende Anbausätze für Operationstische [BLANKO].

Indikationen für die Stabilisierung im Halo-Fixateur externe sind nicht verschobene oder gut reponierte Frakturen und diskoligamentäre Verletzungen der oberen Halswirbelsäule. Eine gute Ausheilung ohne Operation muss erwartet werden. In Einzelfällen ist der Halo-Fixateur auch für Patienten in nicht operablem $\mathrm{Zu}$ stand geeignet.

Der Halo-Fixateur externe wird nach einer gewissen Gewöhnungszeit sehr gut vertragen. Tragezeiten von 6-12 Wochen nach Verletzung sind anzusetzen.

Die Handhabung des Halo-Fixateur-externe muss von Ärzten, Pflegepersonal im OP und Ambulanz und Lagerungspflegern regelmäßig geübt werden.

\section{Operative Behandlung}

\section{Lagerung}

Ob von ventral oder dorsal operiert wird, für die Lagerung gilt, dass der Kopf gut unterstützt, frei ausgelagert wird. Der Kopf wird entweder in einer Schale aufgelegt und befestigt oder mit einem Halo-Ring eingespannt $[2,4,11]$.

Die Lagerung hat 2 Aufgaben:

1. Reposition und vor allem Halten des reponierten Zustandes.

2. Freie Durchleuchtbarkeit mit dem Bildverstärker in 2 Ebenen (Abb. 7a-c).

Wir selbst empfehlen die Abdeckung so vorzunehmen, dass der Bildwandler frei von Tüchern steril durchgeschwenkt werden kann. Wir nennen dies „Mumienabdeckung“, weil der Patient wie eine Mumie steril eingepackt ist. Das Operationsgebiet ist gut zugänglich, der Bildwandler kann unter dem Patienten a. p. und seitlich steril durchgeschwenkt werden. Der Anästhesist und seine Narkosegeräte befinden sich am Fußende (Abb.8). Die etwas längeren Schläuche und Zuleitungen haben keinen Nachteil.

Damit die Schultern nicht stören, ziehen wir diese mit $2-3 \mathrm{~kg}$ belastet über Rollen nach distal. Dieser Effekt kann auch mit Pflasterzügeln erreicht werden. Diese Maßnahmen sind auch bei Operationen

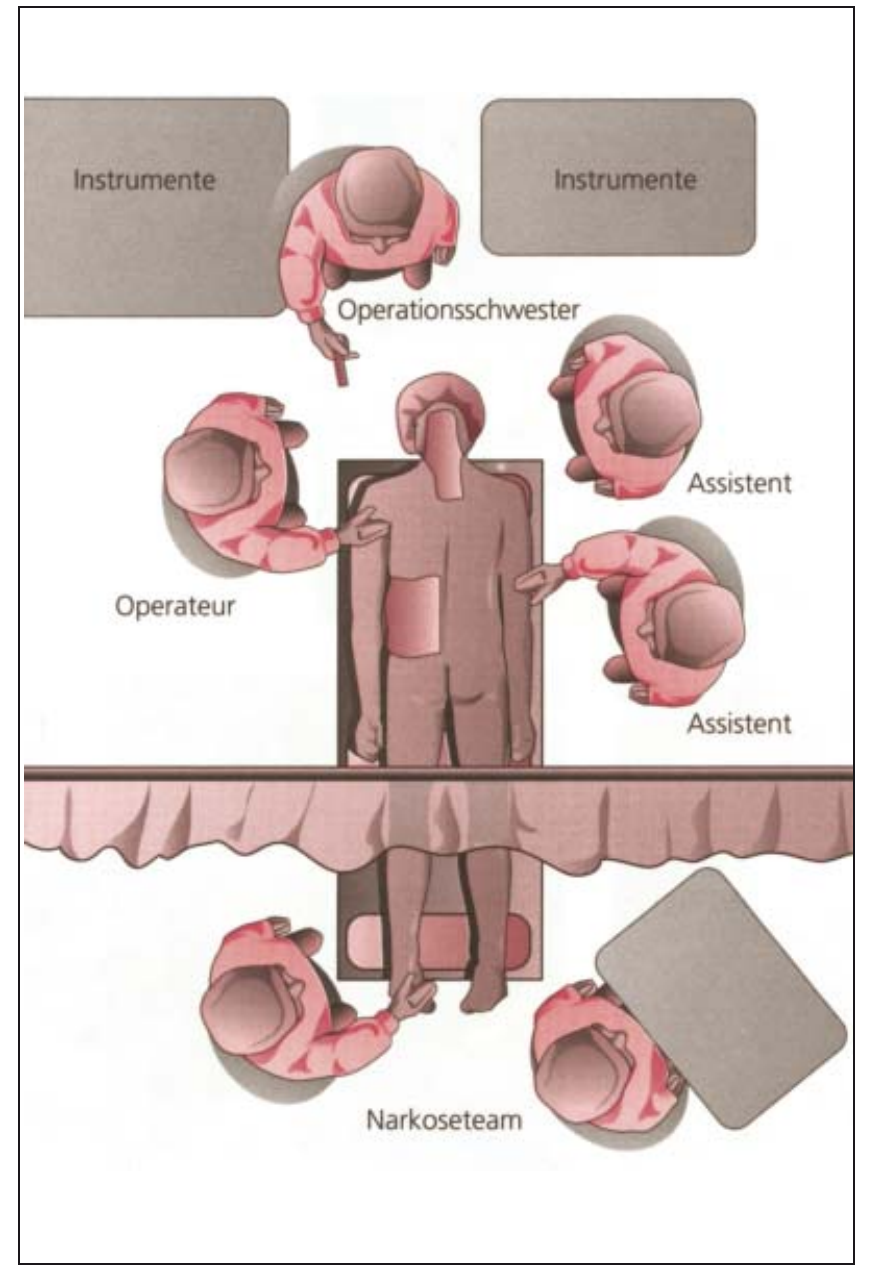

Abb. 8 Stellung von Operateur, Assistenten, Operationsschwester und Narkoseteam bei einer Halswirbelsäulenoperation (in diesem Fall von dorsal ähnlich von ventral) nach Louis [9]. der oberen Halswirbelsäule sinnvoll, aber bei Operationen der unteren Halswirbelsäule ab C5 unbedingt erforderlich.

Die Kontrolle im BV in allen Ebenen muss vor der sterilen Abdeckung überprüft werden. Dies dient einerseits dazu, um das vor der Operation erreichte Repositionsergebnis noch einmal exakt darzustellen und andererseits dazu, dass Operateur und Pflegepersonal die Handhabung und Führung des BVs vor der delikaten Operation beherrschen. So weiß man dann während der Operation genau, wie der BV geführt und geschwenkt werden muss.

Die Lagerung sollte in jedem Haus, welches Operationen an der Halswirbelsäule durchführt, von den beteiligten Ärzten, Lagerungspflegern und Instrumentierenden geübt werden. Dies kann sehr gut gegenseitig geübt werden (Siehe OPJournal). Auf diese Weise kann bei diesen delikaten Operationen bereits präoperativ erhebliches Stresspotenzial abgebaut werden.
Die Lagerung, die Abdeckung und die Handhabung des BV müssen für Operationen an der Halswirbelsäule von Ärzten, Pflegepersonal und Lagerungspflegern regelmäßig geübt werden.

\section{Operationsmethoden}

Die gängigsten Operationsmethoden sind:

- Densverschraubung von vorne

- atlantoaxiale Fusion von dorsal

- dorsale atlantoaxiale Fusion mit transartikulärer Verschraubung (offen und/oder minimalinvasiv) - okzipitozervikale Fusion.

\section{Implantate und Instrumente}

Häufigste Implantate und Instrumente für die operative Versorgung bei Verletzungen der oberen Halswirbelsäule.

- lange Schraubensysteme mit langen Instrumenten für die ventrale Densverschraubung, dorsale transartikuläre C1-/C2-Verschraubung

- die transpedunkuläre C2-Verschraubung 
- Cerclagensysteme für die C1-/C2-Fusion

- Schrauben Platten/Fixateur-interneSysteme wie Cervifix für die längerstreckigen cervikalen Fusionen von dorsal bzw. die okzipitozervikalen Fusionen

- Plattensysteme für occipitocervikale Fusionen von dorsal

- Kombinationen

- beiSpongiosaplastiken, Entnahme am vorderen oder hinteren Beckenkamm als Span und als Späne je nach Vorgabe der Operationsmethode.

Alle Operationsmethoden im Einzelnen hier zu beschreiben, würde diesen Artikel sprengen. Darüber hinaus werden diese sehr ausführlich in der genannten Literatur (s.o.) beschrieben und würden hier nur eine Wiederholung darstellen.

Zur Veranschaulichung und Darstellung soll stellvertretend die Verschraubung von Densfrakturen als eine der häufigsten, auch in nicht Wirbelsäulenzentren durchgeführten Operationen kurz dargestellt werden.

\section{Verschraubung von Densfrakturen (Abb.9)}

Prinzip: Durch eine Zugschraubenosteosynthese wird die Densfraktur unter interfragmentären Druck gesetzt [2,4,5,11].

Indikation: Densfrakturen im Schaftbereich und solche, welche nicht weit in die Basis reichen (d.h. Densfrakturen Anderson II oder III mit geringem Basisanteil).

Operationstechnik: Der präoperativen Lagerung und Reposition kommt die entscheidende Bedeutung zu. Während der Operation kann nicht reponiert werden. Hierzu ist eine erstaunlich wirkende Lordosierung, d.h. Nachhintenneigung des Kopfes erforderlich. Dies muss präoperativ unsteril im Bildwandler in 2 Ebenen vom Operateur kontrolliert werden. Für diesen Vorgang muss genügend Zeit eingeplant werden. Gleichzeitig kann präoperativ der Zugang von Höhe und Richtung der Instrumente simuliert werden. Bei adipösen Patienten und bei Patienten mit Emphysemthorax kann der relativ schräge Zugang der Instrumente wegen des „Berges“ Brustkorb sehr erschwert sein.

- Die Verwendung von 2 Bildwandlern, welche steril abgedeckt in der a.p. und seitlichen Position gehalten werden können, ist sehr hilfreich. Das Einbringen der Zieldrähte sowie der
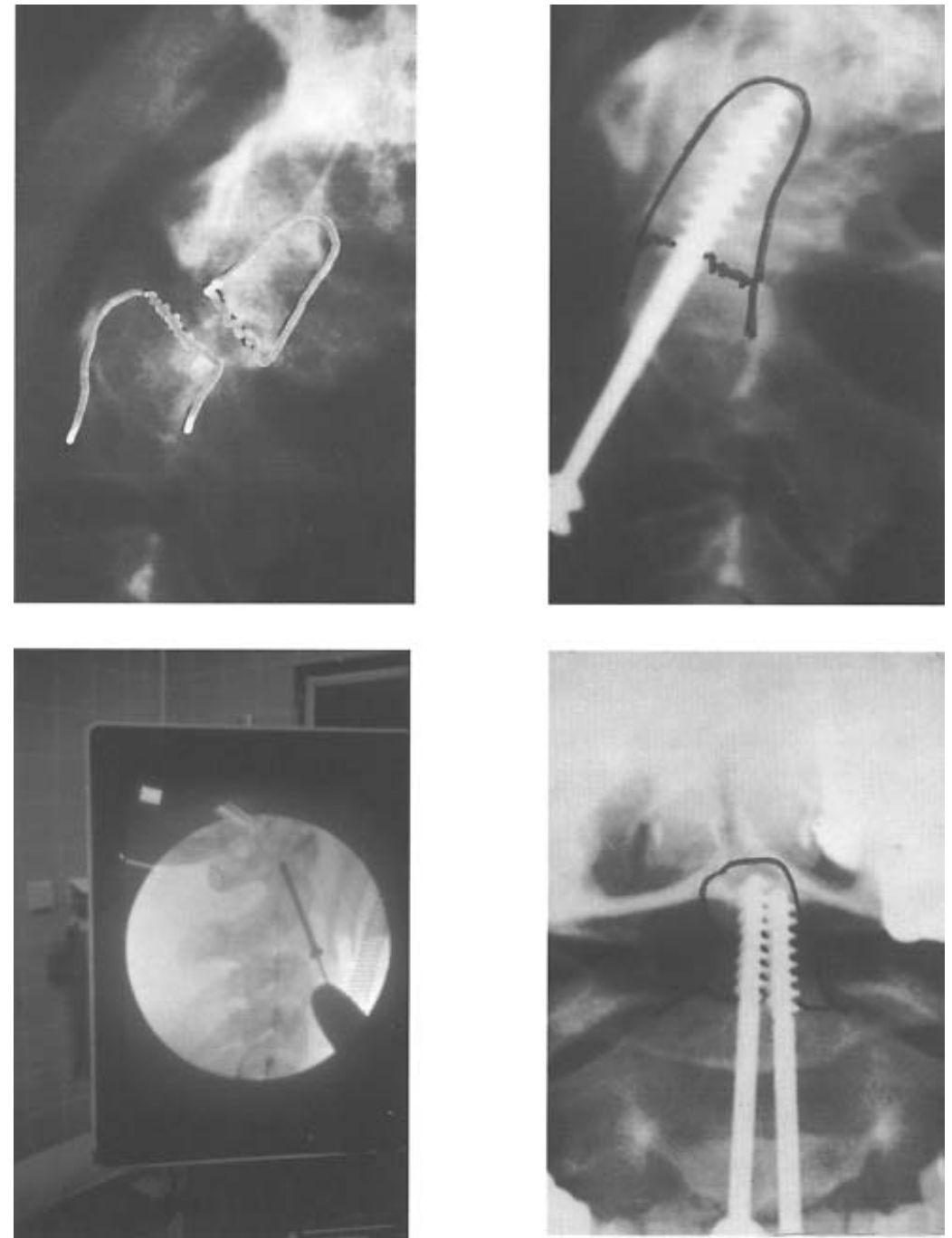

Abb.9 Nachgezeichnete BV-Bilder der Operationsschritte für eine Densverschraubung.

Schrauben, am besten kanülierte Schrauben, muss streng in beiden Bildwandlern sehr engmaschig kontrolliert werden, damit höchstgefährliche Fehllagen von Kirschner-Drähten und Schrauben sowie Vorwärtsschieben der Kirschner-Drähte absolut vermieden wird.

- Der Zugang: Nach Durchtrennung des Platysmas kommt man mit stumpfer Präparation auf die untere Vorderkante des zweiten Halswirbelkörpers. Die Schraubenlage darf keinesfalls auf die Vorderfläche wandern, sondern muss auf der stabilen Unterkante bleiben. Bei einer leichten Verschiebung ist eher eine solche auf die Grundfläche zu tolerieren.

- OP-Schritte: Unter strenger Bildwandlerkontrolle wird ein Kirschner-Draht eingebracht. Es gibt vielfältige Diskussionen, ob eine oder zwei Schrauben verwendet werden sollen $[2,4,5,11]$.
Hier ist nicht der Ort, dies zu entscheiden. Dies liegt in der Hand des Operateurs und seiner Erfahrungen. Bei der Verwendung von 2 Schrauben ist von vornherein darauf zu achten, dass der erste Kirschner-Draht etwas asymmetrisch liegt, damit die zweite Schraube Platz hat.

- Idealerweise werden kanülierte Systeme verwendet. Mit diesen kann der dann exakt sitzende und überprüfte Kirschner-Draht überbohrt und die Schraube eingebracht werden. Die Schraubenlänge ist so zu wählen, muss der Gewindegang an der Spitze der Schraube die Spitze des Dens gerade eben überschreitet. Nur so kann die notwendige Kompressionskraft aufgebaut werden.

- Von Seiten der Instrumentation ist von vornherein darauf zu achten, dass unter Umständen Varianten der Operationstechnik notwendig werden. Hier- 
zu zählt z.B. eine sog. Antigleitplatte, welche angebogen unter den Schraubenkopf gelegt wird, um das Vorgleiten einer weit in den Korpus reichenden Fraktur zu vermeiden.

- Jede „Schule“ hat seine eigenen operationstechnischen Kniffe, welche für diese zwar seltenen, aber um so defizieleren Operationen am besten vorbesprochen wird, damit nicht intraoperativ das Diskutieren und Suchen der geeigneten Implantate und Instrumente beginnt!

- Naht von Platysma und Haut über eine Drainage.

Densverschraubung:

Indikation: Instabile Densfrakturen Anderson II-(III)

Schritte: (Abb.9)

- präop. Reposition und Lagerung und BV-Kontrolle/Übung

- ventraler Zugang von links nach Simulierung von Höhe und Richtung

- nur Darstellen der unteren Vorderkante des HWK II

- unter BV-Kontrolle in 2 Ebenen Platzierung eines oder 2 KirschnerDrähte

von der unteren Vorderkante des HWK II zur Densspitze

- Bohren mit kanüliertem Bohrer (kontinuierliche BV-Kontrolle in 2 Ebenen!)

- kanülierte Spongiosaschraube(n) mit kurzem Gewinde als Zugschrauben

(kontinuierliche Kontrolle im BV in 2 Ebenen)

- Wundverschluss über Drainage

\section{Literatur}

${ }^{1}$ Aebi M, Nazarian S. Klassifikation der Halswirbelsäulenverletzungen. Orthopädie 1987; 16: 27-36

${ }^{2}$ Aebi M, Thalgott JS, Webb JK. AO/ASIF Principles in Spine Surgery. Springer Berlin Heidelberg (1998)

${ }^{3}$ Anderson LD, D'Alonzo RT. Fractures of the odontoid process of the axis. J Bone and Joint Surgery 1974; 56A: $1663-1674$

${ }^{4}$ Blauth M. Obere Halswirbelsäule in Tscherne $\mathrm{H}$, Blauth $\mathrm{M}$. (Hrsg). Tscherne Unfallchirurgie Wirbelsäule Springer Berlin Heidelberg (1998)

${ }^{5}$ Böhler J. Schraubenosteosynthese von Frakturen des Dens axis. Unfallheilkunde 1982; 84: $221-223$

${ }^{6}$ Illgner A, Haas N, Blauth M, Tscherne H. Die operative Behandlung von Verletzungen der Halswirbelsäule. Unfallchirurgie 1989; 92 $363-372$

${ }^{7}$ Illgner A, Reilmann H. Verletzungen der Halswirbelsäule. Unfallchirurg 1996; 99: 351-367

${ }^{8}$ Josten Ch, Russe O, Hahn M, Muhr G. Einteilung der C2-Ringbrüche im Hinblick auf Halo Reposition oder operative Stabilisierung. In: Verletzungen der Wirbelsäule, 28. Jahrestagung der ÖGU (1995)
${ }^{9}$ Louis R. Die Chirurgie der Wirbelsäule. Springer Verlag (1985)

${ }^{10}$ Russe O, Bötel U, Muhr G. Einteilung der C1Ringbrüche nach therapeutischen Gesichtspunkten. In: Verletzungen der Wirbelsäule, 28. Jahrestagung der ÖGU (1995)

${ }^{11}$ Rüedi TP, Murphey WM. AO Principles of Fracture Management. Thieme Stuttgart (2000)

${ }^{12}$ Schwarz N. Die verhakte Rotationssubluxation der Halswirbelsäule. Unfallchirurgie 1992: 95: 367-374

\section{Andreas Badke}

Oberarzt

Prof. Dr. med. Dankward Höntzsch

Leitender Arzt der Abteilung

Medizintechn. Entwicklung

Prof. Dr. med. Hans-Peter Kaps

Chefarzt

Berufsgenossenschaftliche Unfallklinik Schnarrenbergstr. 95

D-72076 Tübingen 